\title{
Eco-friendly Synthesis of 1, 4-Dihydropyrano-[2,3-c] Pyrazoles Using Copper Nanoparticles Grafted on Carbon Microsphere as a Heterogeneous Catalyst
}

\author{
Nitishkumar S. Kaminwar ${ }^{1}$, Sunil. U. Tekale ${ }^{2}$, Anil B. Chidrawar ${ }^{3}$, László Kótai ${ }^{4}$, \\ Rajendra P. Pawar ${ }^{2, * \text { (D) }}$

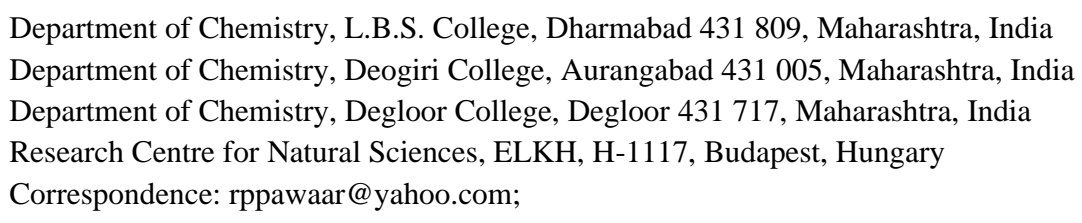

Received: 16.07.2020; Revised: 10.08.2020; Accepted: 12.08.2020; Published: 15.08.2020

\begin{abstract}
A convenient and efficient one-pot three-component synthesis of 6-amino-4-aryl-5-cyano-3methyl-1-phenyl-1, 4-dihydropyrano[2, 3-c] pyrazoles was achieved using copper nanoparticles grafted on carbon microsphere $(\mathrm{Cu}-\mathrm{NP} / \mathrm{C})$ as a reusable heterogeneous catalyst. The synthesis of pyranopyarolzes was carried out under mild reaction conditions affording excellent yield of the corresponding products.
\end{abstract}

Keywords: Pyranopyrazole; Multi-component reaction; $\mathrm{Cu}-\mathrm{NP} / \mathrm{C}$.

(C) 2020 by the authors. This article is an open-access article distributed under the terms and conditions of the Creative Commons Attribution (CC BY) license (https://creativecommons.org/licenses/by/4.0/).

\section{Introduction}

The shift of organic synthesis is prominently directed towards "Greenery". Growing interest awakened among researchers that consequently resulted in the development of new environmentally benign procedures such as the use of the aqueous medium, multi-component reaction (MCR) strategy, and reusable catalysts to save resources and energy [1]. In recent years, the efficient synthesis of complex molecules from simple and readily available materials has become one of the important aspects of organic synthesis. Attempts have been made to develop several techniques for the efficient use of reusable catalysts and multi-component reactions [2-3]. Being powerful and bond-forming efficient tools in organic, combinatorial, and medicinal chemistry, MCRs have attracted researchers with increasing awareness to develop high atom economy, eco-friendly, one-pot, single step, energy minimized, and negligible waste production strategies. These features encourage MCRs as well-suited for the easy construction of diversified arrays of valuable bioactive heterocycles.

Constituting an important heterocyclic scaffold with a rich bioactive profile in medicinal chemistry, dihydropyrano [2, 3-c] pyrazoles exhibit anticancer [4], antimicrobial [5], antioxidant [6], anti-inflammatory [7]. Besides this, they also possess DNA-binding ability [8] and potential inhibitors of cholinesterases [9]. Hence, due to the existence of these precious scaffolds in biologically active molecules (Figure 1), considerable efforts have been documented for the synthesis of these heterocyclic motifs by using various methodologies [10]. From the literature survey, 1,4-dihydro-pyrano[2,3-c]pyrazoles can be synthesized in the 
presence of catalysts like per- 6-amino- $\beta$-cyclodextrin [11], nano- $\mathrm{Fe}_{3} \mathrm{O}_{4}-\mathrm{SiO}_{2},\left(\mathrm{CH}_{2}\right)_{3}-$ imidazole- $\mathrm{SO}_{3} \mathrm{H}$ ] Cl [12], $\mathrm{CsF}$ [13], ionic liquid [bmim] $\mathrm{OH}$ [14], tungstate sulfuric acid [15], HTMAB [16], and SnS-NP [17]. Myrboh et al. reported the synthesis of pyranopyrazoles using L-proline and KF-alumina as a catalyst [18]. Synthesis of pyranopyrazoles using hydrazine hydrate, ethyl acetoacetate, malononitrile, and aromatic aldehydes is also been reported in the presence of catalysts such as $\beta$-cyclodextrin [19], triethylamine [20], cinchona alkaloid derivatives [21], and imidazole [22], meglumine [23], Amberlyst A21 [24], piperidine [25], potassium phthalimide [26], $\mathrm{Ni}_{0.5} \mathrm{Zn}_{0.5} \mathrm{Fe}_{2} \mathrm{O}_{4}$ [27], $\mathrm{Ag} / \mathrm{TiO}_{2}$ [28], isonicotinic acid [29], [Bmim] $\mathrm{BF}_{4}$ and L-Proline [30], CuI nanoparticles [31], 1-(carboxymethyl) pyrinium iodide [cmpyl]I [32]. These reported methodologies have shown good results in many instances. However, some of them also have limitations in terms of the metal catalyst, expensive reagents, long reaction time, environmentally hazardous, harsh reaction conditions, tedious work-up procedure, unsatisfactory yield, and homogeneous catalysts, which are difficult in separation from the reaction mixture.
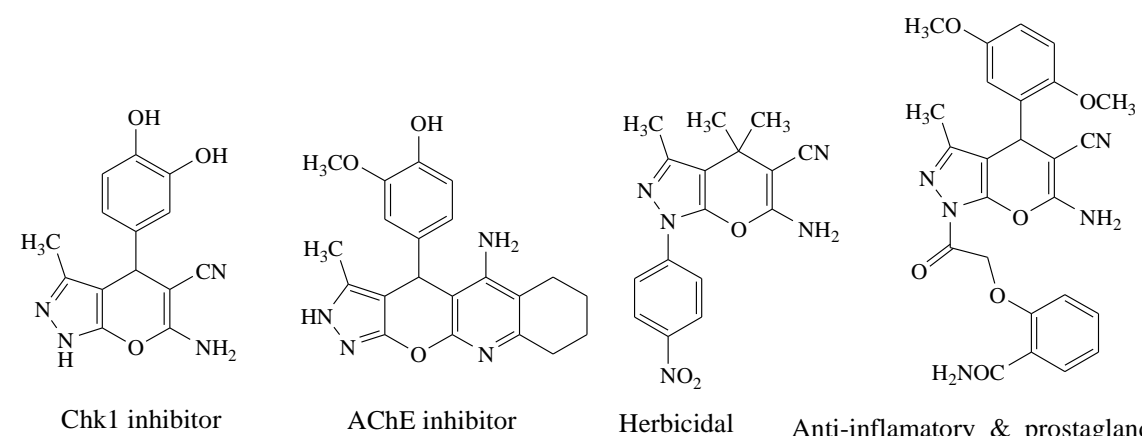

Figure 1. Some biologically active pyranopyrazole derivatives.

Heterogeneous catalysts have gained extensive applications as eco-friendly, economically viable, and easily separable catalysts in organic transformations [33]. Among the heterogeneous catalysts, various nanoparticles (NPs) have received considerable attention for their high catalytic activity and selectivity in various fields of synthetic organic and bioorganic chemistry [34].

Copper nanoparticles are an important group of nano-catalysts. Copper nanoparticle catalyzed reactions are advantageous over the conventional metal-catalyzed reactions in terms of low catalyst loading, high atom economy, better yields, inexpensive, shorter reaction times, and recyclability of the catalyst [35].<smiles>O=Cc1ccccc1</smiles>

(1)

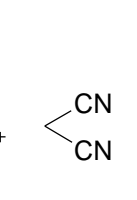

(2)

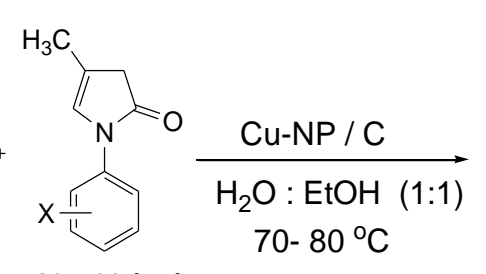

$$
\begin{aligned}
& X=\mathrm{H}(3 a) \\
& X=2-C l(3 b)
\end{aligned}
$$

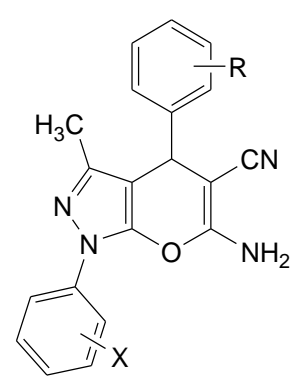

$(4 a-4 n) /(5 a-5 e)$

Scheme 1. One-pot synthesis of pyranopyrazole derivatives.

Nanoparticles may undergo self-aggregation; therefore, it is difficult to separate them from the reaction medium. To avoid the limitation of nanoparticles, they can be supported on some supports. These developed catalytic metallic nanoparticles are inexpensive, non or minimally poisonous, highly active, stable, and easily separable from reaction mixture are 
highly useful. In our previous work, copper nanoparticles grafted on carbon microsphere $(\mathrm{Cu}-$ $\mathrm{NP} / \mathrm{C}$ ) were found to be useful for organic transformations [36-37]. In continuation of our previous work [38-40], herein, we report a new eco-friendly three-component protocol for the synthesis of 1,4-dihydropyrano[2,3-c] pyrazole derivatives using $\mathrm{Cu}$ NP/C as a heterogeneous catalyst in water and ethanol (1:1) solvent (Scheme 1).

\section{Materials and Methods}

\subsection{General.}

All chemicals were purchased from Sigma Aldrich, Alpha Aiser, Spectrochem, and used without further purification. TLC was carried out with Merck silica gel 60-F-254 aluminum plates. ${ }^{1} \mathrm{H}$-NMR and ${ }^{13} \mathrm{C}$-NMR spectra were recorded on a Bruker spectrometer using $\mathrm{CDCl}_{3}, \mathrm{DMSO}_{-} \mathrm{d}_{6}$ as solvent and tetramethylsilane as an internal standard. Mass spectrometric data were recorded by an electron spray ionization (EST) technique on a Q-tofmicro quadruple mass spectrometer (Micro mass). The catalyst synthesis and characterization have been well studied in our previous publications [35-36].

2.2. General procedure for the synthesis of 6-amino-4-aryl-5-cyano-3-methyl-1-phenyl-1, 4dihydropyrano [2, 3-c] pyrazoles.

A mixture of aromatic aldehyde $(1 \mathrm{mmol})$, malononitrile $(1.1 \mathrm{mmol})$, pyrazolin-5-one $(1 \mathrm{mmol})$ in 1:1 water: ethanol $(5 \mathrm{~mL})$ in the presence of $\mathrm{Cu}-\mathrm{NP} / \mathrm{C}(10 \mathrm{mg})$ was refluxed for an appropriate period of time. The progress of the reaction was monitored by TLC. After completion of the reaction, the mixture was cooled to room temperature and washed with ethanol. The product and catalyst were separated by filtration. The product was used without further purification methods, and the catalyst was washed with ethyl acetate, dried, and reused.

\subsection{Spectral data.}

6-Amino-3-methyl-4-(3-chlorophenyl)-2,4-dihydropyrano[2,3-c]pyrazole-5-carbonitrile, entry 3, (4c): Melting point $=181-183^{\circ} \mathrm{C}$; IR $\left(\mathrm{cm}^{-1}\right) 783,831,921,955,1062,1182,1242$, 1294, 1394, 1435, 1537, 1627, 1820, 1896, 2017, 2185, 2569, 2777, 2895, 2964, 3070, 3315, 3421; ${ }^{1} \mathrm{H}$ NMR (300 MHz, DMSO-d6): $\delta$ ppm H (ppm) 1.910 (s, 3H, CH3), 4.649 (s, $1 \mathrm{H}$ chiral),

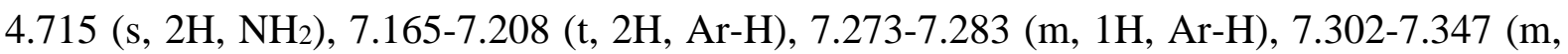
2H, Ar-H), 7.451-7.491 (t, 2H, Ar-H), 7.641-7.659 (d, 2H, Ar-H) ; ${ }^{13} \mathrm{C}$ NMR $(500 \mathrm{MHz}$, DMSO d6): $\delta$ ppm 78.05, 154.21, 161.52, 174.45; HRMS : 363.

6-Amino-4-(4-methoxyphenyl)-3-methyl-2,4-dihydropyrano[2,3-c]pyrazole-5-carbonitrile, entry 10, (4j): Melting point $=174-175^{\circ} \mathrm{C}$; IR $\left(\mathrm{cm}^{-1}\right)$ 837, 962, 1026, 1068, 1107, 1128, 1174 , 1253, 1301, 1336, 1390, 1456, 1514, 1587, 1656, 1894, 2189, 2843, 2920, 2968, 3201, 3267, 3317, 3387; ${ }^{1} \mathrm{H}$ NMR (300 MHz, DMSO d6): $\delta$ ppm 1.898 (s, 3H, CH3), 3.808 (s, 3H, OCH $)$, 4.601 (s, 1H, chiral), 4.692 (s, 2H, NH2), 6.869-6.890 (d, 2H, Ar-H), 7.159-7.181 (t, 2H, ArH), 7.298-7.333 (t, 1H, ArH), 7.444-7.484 (t, 2H, ArH), 7.643-7.665(d, 2H, ArH); HRMS : 359.

6-Amino-1-(2-chlorophenyl)-1,4-dihydro-3-methyl-4-(3-nitrophenyl)pyrano[2,3-c]pyrazole5-carbonitrile, entry 17, (5c): Melting point $=199-201^{\circ} \mathrm{C} ; \mathrm{IR}\left(\mathrm{cm}^{-1}\right) 889,929,1041,1114$, 1184, 1251, 1269, 1350, 1392, 1475, 1496, 1585, 1656, 2187, 2868, 2976, 3190, 3309, 4315; ${ }^{1} \mathrm{H}$ NMR (300 MHz, DMSO-d6): $\delta$ ppm 1.796 (s, 3H, CH3), 4.989 (s, 1H chiral), 7.222 (s, 2H, $\mathrm{NH}_{2}$ ), 7.522-7.579 (m, 2H, Ar-H), 7.661-7.751 (m, 4H, Ar-H), 8.133-8.179 (t, $\left.2 \mathrm{H}, \mathrm{Ar}-\mathrm{H}\right) ;{ }^{13} \mathrm{C}$ 
NMR (500 MHz, DMSO d6): $\delta$ (ppm) 12.44, 96.15, 119.61, 122.03, 122.15, 128.19, 129.88, $130.13,130.25,130.82,131.14,133.96,134.40,145.15,145.38,146.02,147.88,159.58$; HRMS : 408.07.

\section{Results and Discussion}

Due to growing interest in solid catalysts for the synthesis of heterocycles [34], herein we describe an efficient and facile protocol for the synthesis of 1,4-dihydropyrano[2,3c]pyrazole derivatives from aromatic aldehydes (1a-n), malononitrile (2) and 3-methyl-1phenyl-2-pyrazolin-5-one (3a-b) using $\mathrm{Cu} \mathrm{NP} / \mathrm{C}$ as a reusable and safe catalyst in water-ethanol $(1: 1)$ at $70-80{ }^{\circ} \mathrm{C}$ (Scheme 1). The present work was performed by using this catalyst for the synthesis of pyranopyrazole derivatives. $\mathrm{Cu}$ NP/C was collected by a simple work-up procedure and could be reused several times without appreciable loss of catalytic activity. Another aspect of this work is the use of water:ethanol (1:1) system as a solvent.

Initially, p-anisaldehyde (1 mmol), malononitrile $(1.1 \mathrm{mmol})$, and 3-methyl 1-phenyl 5-pyrazolone ( $1 \mathrm{mmol}$ ) were selected for the optimization of the reaction conditions at 70-80 ${ }^{\circ} \mathrm{C}$ in $5 \mathrm{~mL}$ water-ethanol (1:1). Firstly, in the absence of the catalyst, only a $25 \%$ yield of the desired product was obtained at $70-80^{\circ} \mathrm{C}$. Then, to identify the suitable reaction condition, the effect of solvent and amount of catalyst was studied. The high yield was obtained using $10 \mathrm{mg}$ of the catalyst in 1:1 $\mathrm{H}_{2} \mathrm{O}-\mathrm{EtOH}$ (Table 1).

Table 1. Optimization of reaction conditions.

\begin{tabular}{|c|c|c|c|c|c|}
\hline Entry & Solvent & Catalyst (mg) & Temperature $\left({ }^{\circ} \mathrm{C}\right)$ & $\begin{array}{l}\text { Time } \\
\text { (min.) }\end{array}$ & $\begin{array}{c}\text { Yield } \\
(\%)\end{array}$ \\
\hline 1 & $\mathrm{EtOH}$ & - & $70-80$ & $22 \mathrm{hr}$ & 25 \\
\hline 2 & $\mathrm{CH}_{3} \mathrm{CN}$ & 10 & $80-90$ & 100 & 60 \\
\hline 3 & $\mathrm{CH}_{2} \mathrm{Cl}_{2}$ & 10 & 40 & 140 & 65 \\
\hline 4 & $\mathrm{H}_{2} \mathrm{O}$ & 05 & $80-90$ & 110 & 65 \\
\hline 5 & EtOH & 05 & $70-80$ & 90 & 70 \\
\hline 6 & $\mathrm{H}_{2} \mathrm{O}: \mathrm{EtOH}(1: 1)$ & 05 & $70-80$ & 100 & 60 \\
\hline 7 & $\mathrm{H}_{2} \mathrm{O}: \mathrm{EtOH}(1: 1)$ & 10 & $70-80$ & 15 & 93 \\
\hline 8 & $\mathrm{H}_{2} \mathrm{O}: \mathrm{EtOH}(1: 1)$ & 15 & $70-80$ & 17 & 90 \\
\hline
\end{tabular}

Afterward, a series of reactions were carried out by using diversely substituted aldehydes under the optimized reaction conditions. All the aldehydes underwent a threecomponent reaction with malononitrile and 3-methyl-1-phenyl-2-pyrazolin-5-one to produce a library of 1, 4-dihydropyrano [2, 3-c]pyrazole derivatives in good to excellent yields (Table 2). The reactions of aromatic aldehydes with electron-withdrawing groups such as chloro, nitro, proceeded at faster rates and gave better yields than those with electron-donating groups such as methoxy and methyl.

Table 2. Synthesis of 6-amino-4-aryl-5-cyano-3-methyl-1-phenyl-1, 4-dihydropyrano [2, 3-c] pyrazolescatalyzed by $\mathrm{Cu}-\mathrm{NP} / \mathrm{C}$.

\begin{tabular}{|c|c|c|c|c|c|c|}
\hline Entry & Aldehyde & Enolizable Ketone & Time (min.) & Product & Yield $^{\mathrm{b}}(\%)$ & $\mathbf{M P}\left({ }^{\circ} \mathbf{C}\right)$ \\
\hline 1 & Benzaldehyde & $3 \mathrm{a}$ & 20 & $4 \mathrm{a}$ & 86 & $171-173^{15}$ \\
\hline 2 & 2-Chloro benzaldehyde & $3 a$ & 18 & $4 \mathrm{~b}$ & 88 & $145-146^{15}$ \\
\hline 3 & 3-Chloro benzaldehyde & $3 \mathrm{a}$ & 20 & $4 \mathrm{c}$ & 90 & $158-160^{16}$ \\
\hline 4 & 3-Bromo benzaldehyde & $3 a$ & 17 & $4 d$ & 90 & $159-160^{15}$ \\
\hline 5 & 3-Nitro benzaldehyde & $3 a$ & 15 & $4 \mathrm{e}$ & 88 & $190-192^{15}$ \\
\hline 6 & 4-Fluoro benzaldehyde & $3 a$ & 17 & $4 \mathrm{f}$ & 90 & $163-164^{13}$ \\
\hline 7 & 4-Nitro benzaldehyde & $3 a$ & 15 & $4 \mathrm{~g}$ & 93 & $195-197^{15}$ \\
\hline 8 & 4-Bromo benzaldehyde & $3 a$ & 18 & $4 \mathrm{~h}$ & 88 & $182-184^{15}$ \\
\hline 9 & 4-Tolualdehyde & $3 a$ & 24 & $4 \mathrm{i}$ & 85 & $167-168^{15}$ \\
\hline 10 & 4-Anisaldehyde & $3 a$ & 30 & $4 j$ & 84 & $174-175^{15}$ \\
\hline 11 & 4-Cyano benzaldehyde & $3 a$ & 16 & $4 \mathrm{k}$ & 91 & $218-220^{17}$ \\
\hline
\end{tabular}




\begin{tabular}{l|l|l|l|l|l|l}
\hline Entry & Aldehyde & Enolizable Ketone & Time (min.) & Product & Yield ${ }^{\mathbf{b}}(\boldsymbol{\%})$ & MP $\left({ }^{\mathbf{O}} \mathbf{C}\right)$ \\
\hline 12 & 4-Hydroxy benzaldehyde & $3 \mathrm{a}$ & 24 & 41 & 85 & $211-213^{16}$ \\
\hline 13 & Terphthaldehyde & $3 \mathrm{a}$ & 25 & $4 \mathrm{~m}$ & 90 & $238-240^{12}$ \\
\hline 14 & 3,4-Dimethoxy benzaldehyde & $3 \mathrm{a}$ & 36 & $4 \mathrm{n}$ & 86 & $192-194^{13}$ \\
\hline 15 & 4-Fluoro benzaldehyde & $3 \mathrm{~b}$ & 24 & $5 \mathrm{a}$ & 88 & $173-175$ \\
\hline 16 & $\begin{array}{l}\text { 4-Dimethylamino } \\
\text { benzaldehyde }\end{array}$ & $3 \mathrm{~b}$ & 28 & $5 \mathrm{~b}$ & 85 & $168-170$ \\
\hline 17 & 3-Nitro benzaldehyde & $3 \mathrm{~b}$ & 20 & $5 \mathrm{c}$ & 91 & $199-201$ \\
\hline 18 & 3-Chloro benzaldehyde & $3 \mathrm{~b}$ & 22 & $5 \mathrm{~d}$ & 90 & $162-164$ \\
\hline 19 & 5-Bromo salicyaldehyde & $3 \mathrm{~b}$ & 26 & $5 \mathrm{e}$ & 88 & $212-214$
\end{tabular}

aAldehyde (1 mmol), malononitrile (1mmol), enolizable ketone (1 mmol) and Cu NP/C $10 \mathrm{mg}, \mathrm{H}_{2} \mathrm{O}: \mathrm{EtOH}(1: 1)$

$5 \mathrm{~mL}$, reflux; ' ${ }^{\mathrm{b}}$ Isolated yield.

The mechanism of the $\mathrm{Cu}-\mathrm{NP} / \mathrm{C}$ catalyzed synthesis of pyranopyarazoles has been depicted in Figure 2. Initially, the catalyst promotes the formation of arylidene malononitrile by the Knoevenagel condensation between aldehyde and malononitrile. Simultaneously there is the activation of the methylene group of pyrazolone, which combines with the arylidene malononitrile followed by cyclization, and tautomerization finally results in the formation of the desired product.

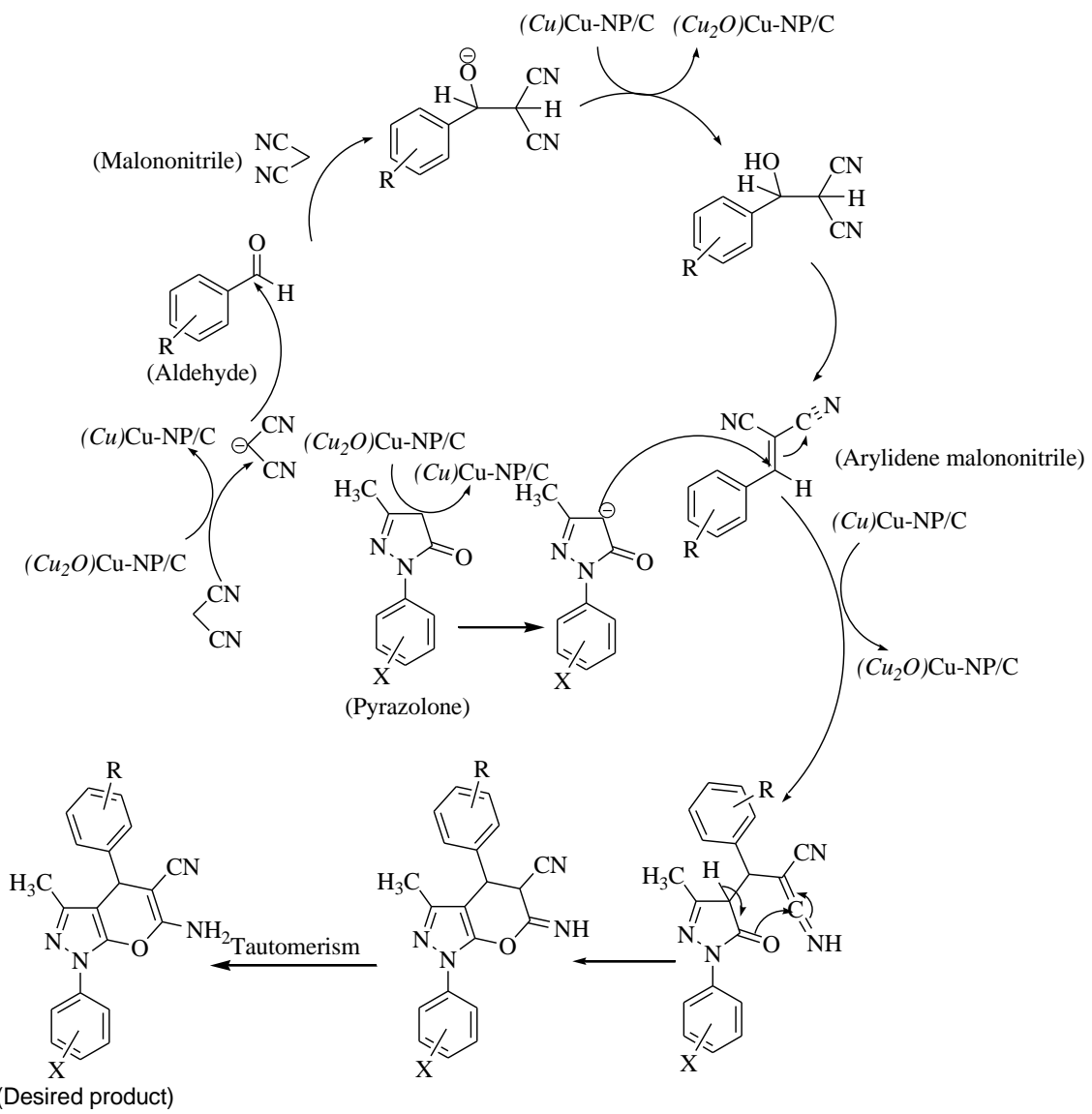

Figure 2. Mechanism of $\mathrm{Cu}-\mathrm{NP} / \mathrm{C}$ catalyzed synthesis of pyranopyarazoles.

\section{Conclusions}

In summary, we report an efficient one-pot three-component synthesis of 6-amino-4aryl-5-cyano-3-methyl-1-phenyl-1,4-dihydropyrano [2, 3-c] pyrazoles by the reaction of various aldehydes, malononitrile and pyrazolin-5-one using copper nanoparticles grafted on carbon microsphere as a reusable heterogeneous catalyst. Use of easily separable $\mathrm{Cu}-\mathrm{NP} / \mathrm{C}$ as a heterogeneous catalyst, non-hazardous reaction conditions, clean reaction conditions, and the 
use of aqueous ethanol as the reaction solvent makes the present protocol an environmentally benign and green approach for the synthesis of dihydropyranopyrazoles.

\section{Funding}

This research received no external funding.

\section{Acknowledgments}

The authors are thankful to the Principal L.B.S. College, Dharmabad, and also to the Principal, Deogiri College, Aurangabad.

\section{Conflicts of Interest}

The authors declare no conflict of interest.

\section{References}

1. Singh, K.N. Multicomponent reactions: A sustainable tool to 1, 2-and 1, 3-azoles. Org. \& Biomol. Chem. 2018, 16, 9084-9116, https://doi.org/10.1039/C8OB01872C.

2. Feng, L.; Wang, K.-Y.; Day, G.S.; Zhou, H.-C. The chemistry of multi-component and hierarchical framework compounds. Chem. Soc. Rev. 2019, 48, 4823-4853, https://doi.org/10.1039/C9CS00250B.

3. Xu, W.; Thapa, K.B.; Ju, Q.; Fang, Z.; Huang, W. Heterogeneous catalysts based on mesoporous metalorganic frameworks. Coord. Chem. Rev. 2018, 373, 199-232, https://doi.org/10.1016/j.ccr.2017.10.014.

4. Vasava, M.S.; Bhoi, M.N.; Rathwa, S.K.; Shetty, S.S.; Patel, R.D.; Rajani, D.P.; Rajani, S.D.; Patel, A.; Pandya, H.A.; Patel, H.D. Novel 1, 4-dihydropyrano [2, 3-c] pyrazole derivatives: Synthesis, characterization, biological evaluation and in silico study. J. Mol. Stru. 2019, 1181, 383-402, https://doi.org/10.1016/j.molstruc.2018.12.053.

5. Reddy, G.M.; Sravya, G.; Yuvaraja, G.; Camilo, A.; Zyryanov, G.V.; Garcia, J.R. Highly functionalized pyranopyrazoles: synthesis, antimicrobial activity, simulation studies and their structure activity relationships (SARs). Res. Chem. Int. 2018, 44, 7491-7507, https://doi.org/10.1007/s11164-018-3569-8.

6. Silva, V.L.; Elguero, J.; Silva, A.M. Current progress on antioxidants incorporating the pyrazole core. Eur. J. Med. Chem. 2018, 156, 394-429, https://doi.org/10.1016/j.ejmech.2018.07.007.

7. Chavan, R.R.; Hosamani, K.M. Microwave-assisted synthesis, computational studies and antibacterial/antiinflammatory activities of compounds based on coumarin-pyrazole hybrid. Royal Soc. Open Sci. 2018, 5, https://doi.org/10.1098/rsos.172435.

8. Badr, M.H.; Abd El Razik, H.A. 1, 4-Disubstituted-5-hydroxy-3-methylpyrazoles and some derived ring systems as cytotoxic and DNA binding agents. Synthesis, in vitro biological evaluation and in silico ADME study. Med. Chem. Res. 2018, 27, 442-457, https://doi.org/10.1007/s00044-017-2071-y.

9. Derabli, C.; Boualia, I.; Abdelwahab, A.B.; Boulcina, R.; Bensouici, C.; Kirsch, G.; Debache, A. A cascade synthesis, in vitro cholinesterases inhibitory activity and docking studies of novel Tacrine-pyranopyrazole derivatives. Bioorg. \& Med. Chem. Lett. 2018, 28, 2481-2484, https://doi.org/10.1016/j.bmcl.2018.05.063.

10. Mamaghani, M.; Nia, R.H. A Review on the recent multi-component synthesis of pyranopyrazoles. Polycyclic Aromatic Compounds 2019, 1-69, https://doi.org/10.1080/10406638.2019.1584576.

11. Kanagaraj, K.; Pitchumani, K. Solvent-free multi-component synthesis of pyranopyrazoles: per-6-amino- $\beta$ cyclodextrin as a remarkable catalyst and host. Tetrahedron Lett. 2010, 51, 3312-3316, https://doi.org/10.1016/j.tetlet.2010.04.087.

12. Zolfigol, M.A.; Ayazi-Nasrabadi R.; Baghery S.; Khakyzadeh V.; Azizian S. Applications of a novel nano magnetic catalyst in the synthesis of 1,8-dioxo-octahydroxanthene and dihydropyrano [2, 3-c] pyrazole derivatives. J. Mol. Cat. A: Chem. 2016, 418, 54-67, https://doi.org/10.1016/j.molcata.2016.03.027.

13. Bhosale, V.N.; Angulwar, J.A.; Khansole, G.S.; Waghmare, G.S. One-pot three-component condensation for the synthesis of 1, 4-dihydropyrano [2,3-c] pyrazoles using cesium fluoride as an efficient catalyst. $J$. Chem. Pharm. Res. 2014, 6, 733-737.

14. Khurana, J.M.; Chaudhary, A. Efficient and Green Synthesis of 4 H-pyrans and 4 H-pyrano [2, 3-c] Pyrazoles Catalyzed by Task-specific Ionic Liquid [bmim] OH under Solvent-free Conditions. Green Chem. Lett. \& Rev. 2012, 5, 633-638, https://doi.org/10.1080/17518253.2012.691183.

15. Mahnaz, F.; Bahador, K.; Iman, S.; Hamideh, M.T. An environmentally friendly synthesis of 1,4dihydropyrano[2,3-c]pyrazole derivatives catalyzed by tungstate sulfuric acid. Chin. Chem. Lett. 2014, 25, 1580-1882, https://doi.org/10.1016/j.cclet.2014.07.012. 
16. Jin, T.S.; Wang, A.Q.; Cheng, Z.L.; Zhang, J.S.; Li, T.S. A clean and simple synthesis of 6-amino-4-aryl-5cyano-3-methyl-1-phenyl-1, 4-dihydropyrano [2, 3-c] pyrazole in water. Synth. Comm. 2005, 35, 137-143, https://doi.org/10.1081/SCC-200046527.

17. Iravani, N.; Keshavarz, M.; Kish, H.A., Parandvar, R. Tin sulfide nanoparticles supported on activated carbon as an efficient and reusable Lewis acid catalyst for three-component one-pot synthesis of $4 \mathrm{H}-$ pyrano [2, 3-c] pyrazole derivatives. Chin. J. Cat. 2015, 36, 626-633, https://doi.org/10.1016/S18722067(14)60284-9.

18. Mecadon, H.; Rohman, M.R.; Rajbangshi, M.; Myrboh, B. $\gamma$-Alumina as a recyclable catalyst for the fourcomponent synthesis of 6-amino-4-alkyl/aryl-3-methyl-2,4-dihydropyrano[2,3-c]pyrazole-5-carbonitriles in aqueous medium. Tetrahedron Lett. 2011, 52, 2523-2326, https://doi.org/10.1016/j.tetlet.2011.03.036.

19. Tayade, Y.A.; Padvi, S.A.; Wagh, Y.B.; Dalal, D.S. $\beta$-Cyclodextrin as a supramolecular catalyst for the synthesis of dihydropyrano[2,3-c]pyrazole and spiro[indoline-3,4'-pyrano[2,3-c]pyrazole] in aqueous medium. Tetrahedron Lett. 2015, 56, 2441-2447, https://doi.org/10.1016/j.tetlet.2015.03.084.

20. Shestopalov, A.M.; Emeliyanova, Y.M.; Shestopalov, A.A.; Rodinovskaya, L.A.; Niazimbetova, Z.I.; Evans, D.H. Cross-condensation of derivatives of cyanoacetic acid and carbonyl compounds. Part 1: Singlestage synthesis of 1'-substituted 6-amino-spiro-4-(piperidine-4')-2H, 4H-pyrano [2, 3-c] pyrazole-5carbonitriles. Tetrahedron 2003, 59, 7491-7496, https://doi.org/10.1016/S0040-4020(03)01178-5.

21. Gogoi, S.; Zhao, C.-G. Organocatalyzed enantioselective synthesis of 6-amino-5-cyanodihydropyrano [2, 3c] pyrazoles. Tetrahedron Lett. 2009, 50, 2252-2255, https://doi.org/10.1016/j.tetlet.2009.02.210.

22. Siddekha, A.; Nizam, A.; Pasha, M.A. An efficient and simple approach for the synthesis of pyranopyrazoles using imidazole (catalytic) in aqueous medium, and the vibrational spectroscopic studies on 6-amino-4-(4'methoxyphenyl)-5-cyano-3-methyl-1-phenyl-1, 4-dihydropyrano [2, 3-c] pyrazole using density functional theory. Spectrochimica Acta A: Mol. \& Biomol. Spectro. 2011, 81, 431-440, https://doi.org/10.1016/j.saa.2011.06.033.

23. Guo, R.Y.; An, Z.M.; Mo, L.P.; Yang, S.T.; Liu, H.X.; Wang, S.X.; Zhang, Z.H. Meglumine promoted onepot, four-component synthesis of pyranopyrazole derivatives. Tetrahedron 2013, 69, 9931-9938, https://doi.org/10.1016/j.tet.2013.09.082.

24. Bihani, M.; Bora, P.P.; Bez, G.; Askari, H. Amberlyst A21 catalyzed chromatography-free method for multicomponent synthesis of dihydropyrano [2, 3-c] pyrazoles in ethanol. ACS Sustainable Chem. \& Eng. 2013, 1, 440-447, https://doi.org/10.1021/sc300173z.

25. Vasuki, G.; Kumaravel, K. Rapid four-component reactions in water: synthesis of pyranopyrazoles. Tetrahedron Lett. 2008, 49, 5636-5638, https://doi.org/10.1016/j.tetlet.2008.07.055.

26. Kiyani, H.; Bamdad, M. One-pot four-component synthesis of 1, 4-dihydropyrano [2, 3-c] pyrazole-5carbonitriles catalyzed by potassium phthalimide. Rev. Roum. Chim. 2017, 62, 221-226.

27. Moeinpour, F.; Khojastehnezhad, A. Cesium carbonate supported on hydroxyapatite coated $\mathrm{Ni}_{0.5} \mathrm{Zn}_{0.5} \mathrm{Fe}_{2} \mathrm{O}_{4}$ magnetic nanoparticles as an efficient and green catalyst for the synthesis of pyrano [2, 3-c] pyrazoles. Chin. Chem. Lett. 2015, 26, 575-579, https://doi.org/10.1016/j.cclet.2015.01.033.

28. Fatahpour, M.; Sadeh, F.N.; Hazeri, N. Maghsoodlou, M.T.; Hadavi, M.S.; Mahnaei, S. Ag/TiO 2 nano-thin films as robust heterogeneous catalyst for one-pot, multi-component synthesis of bis (pyrazol-5-ol) and dihydropyrano [2, 3-c] pyrazole analogs. J. Saudi Chem. Soc. 2017, 21, 998-1006, https://doi.org/10.1016/j.jscs.2017.05.009.

28. Zolfigol, M.A.; Tavasoli, M.; Moosavi-Zare, A.R.; Moosavi, P.; Kruger, H.G.; Shiri, M.; Khakyzadeh, V. Synthesis of pyranopyrazoles using isonicotinic acid as a dual and biological organocatalyst. RSC Advances 2013, 3, 25681-25685, https://doi.org/10.1039/C3RA45289A.

29. Khurana, J.M.; Nand, B.; Kumar, S. Rapid synthesis of polyfunctionalized pyrano [2, 3-c] pyrazoles via multi-component condensation in room-temperature ionic liquids. Synth. Commun. 2011, 41, 405-410, https://doi.org/10.1080/00397910903576669.

30. Safaei-Ghomi, J.; Ziarati A.; Tamimi, M. A Novel method for the one-pot five-component synthesis highly functionalized pyranopyrazoles catalyzed by CuI nanoparticles. Acta Chimica Slov. 2013, 60, 403-410.

31. Moosavi-Zare, A.R.; Zolfigol, M.A.; Salehi-Moratab, R.; Noroozizadeh, E. Catalytic application of 1(carboxymethyl) pyridinium iodide on the synthesis of pyranopyrazole derivatives. J. Mol. Cat. A: Chem. 2016, 415, 144-150, https://doi.org/10.1016/j.molcata.2016.02.003.

32. Corma, A.; Garcia, H. Organic reactions catalyzed over solid acids. Catalysis Today 1997, 38, 257-308, https://doi.org/10.1016/S0920-5861(97)81500-1.

33. Scheuermann, G.M.; Rumi, L.; Steurer, P.; Bannwarth, W.; Mülhaupt, R. Palladium nanoparticles on graphite oxide and its functionalized graphene derivatives as highly active catalysts for the Suzuki-Miyaura coupling reaction. J. Am. Chem. Soc. 2009, 131, 8262-8270, https://doi.org/10.1021/ja901105a.

34. Ojha, N.K.; Zyryanov, G.V.; Majee, A.; Charushin, V.N.; Chupakhin, O.N.; Santra, S. Copper nanoparticles as inexpensive and efficient catalyst: A valuable contribution in organic synthesis. Coord. Chem. Rev. 2017, 353, 1-57, https://doi.org/10.1016/j.ccr.2017.10.004.

35. Pasinszki, T.; Krebsz, M.; Lajgut, G.G.; Kocsis, T.; Kótai, L.; Kauthale, S.; Tekale, S.; Pawar, R. Copper nanoparticles grafted on carbon microspheres as novel heterogeneous catalysts and their application for the 
reduction of nitrophenol and one-pot multi-component synthesis of hexahydroquinolines. NJC 2018, 42, 1092-1098, https://doi.org/10.1039/C7NJ03562D.

36. More, Y.W.; Tekale, S.U.; Kaminwar, N.S.; Kótai, L.; Pasinszki, T.; Kendrekar, P.S.; Pawar, R.P. Synthesis of 3,4-dihydropyrano[c]chromenes using carbon microsphere supported copper nanoparticles (Cu-NP/C) prepared from loaded cation exchange resin as a catalyst. Current Org. Synth. 2019, 16, 288-293, https://doi.org/10.2174/1570179415666181116104931.

37. Khandare, P.M.; Ingale, R.D.; Taware, A.S.; Shisodia, S.U.; Pawar, S.S.; Kotai, L.; Pawar, R.P. One pot synthesis and biological evaluation of pyranopyrazoles in aqueous medium. Eur. Chem. Bull. 2017, 6, 410414, https://doi.org/10.17628/ecb.2017.6.410-414.

38. Rupnar, B.D.; Pagore, V.P.; Tekale, S.U.; Shisodia, S.U.; Pawar, R.P. L-Tyrosine catalysed mild and efficient synthesis of dihydropyrano [2, 3c] pyrazole under microwave irradiation. Der Chemica Sinica 2017, 8, 229-234.

39. Tekale, S.U., Kauthale, S.S., Jadhav, K.M.; Pawar, R.P. Nano-ZnO catalyzed green and efficient one-pot four-component synthesis of pyranopyrazoles. J. Chem. 2013, 2013, 1-8, https://doi.org/10.1155/2013/840954. 